Supporting Information for

\title{
Polar Copolymerization by Pd-Diimine Based Catalyst. Influence of Catalyst Charge and Polar Substituent on Catalyst Poisoning and Polymerization Activity. A Density Functional Theory Study
}

Miklos J. Szabo, ${ }^{\mathrm{a}}$ Richard F. Jordan ${ }^{\mathrm{b}}$, Artur Michalak ${ }^{\mathrm{C}}$, Warren E. Piers ${ }^{\mathrm{a}}$, Thomas Weiss $^{\mathrm{d}}$, Sheng-Yong Yang ${ }^{\mathrm{a}}$ and Tom Ziegler ${ }^{\mathrm{a}}, *$

a Department of Chemistry, University of Calgary, University Dr. 2500, Calgary, AB, Canada T2N 1N4; ${ }^{b}$ Department of Chemistry, 5735 South Ellis Avenue, The University of Chicago, Chicago, Illinois 60637, USA; ' Department of Theoretical Chemistry, Faculty of Chemistry, Jagiellonian University, R. Ingardena 3, 30-060 Cracow, Poland and ${ }^{d}$ Bayer AG, Bayer Polymers, BPO-IIS-INN-SYR-SPE , 51368 Leverkusen, Q18. * ziegler@ucalgary.ca

Content: The optimized geometries of the crucial structures reported in the paper (the atomic symbols followed by three Cartesian Coordinates, in $\AA$ ). The structure labels correspond to those of Scheme 2-4, Table 1-2. 

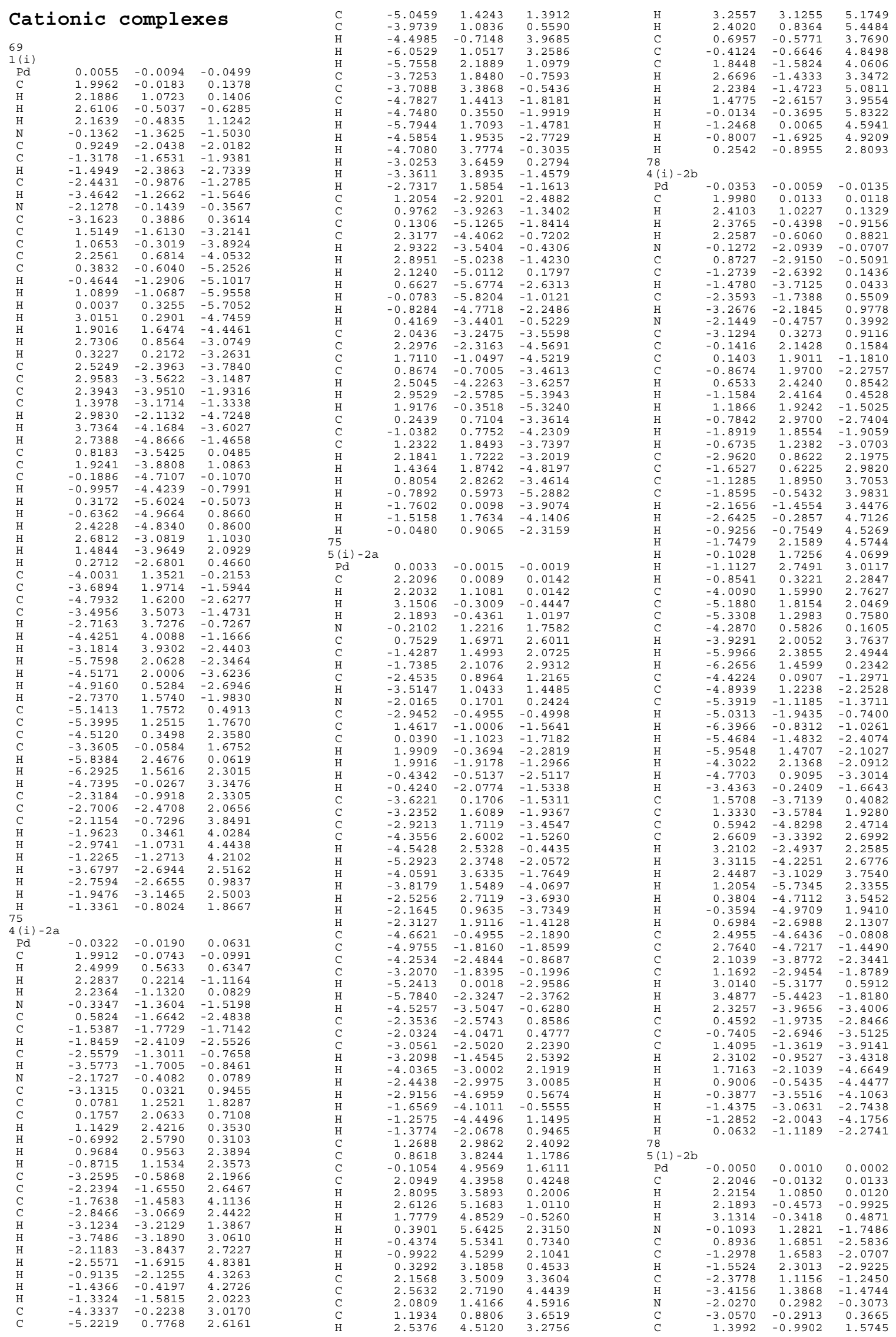

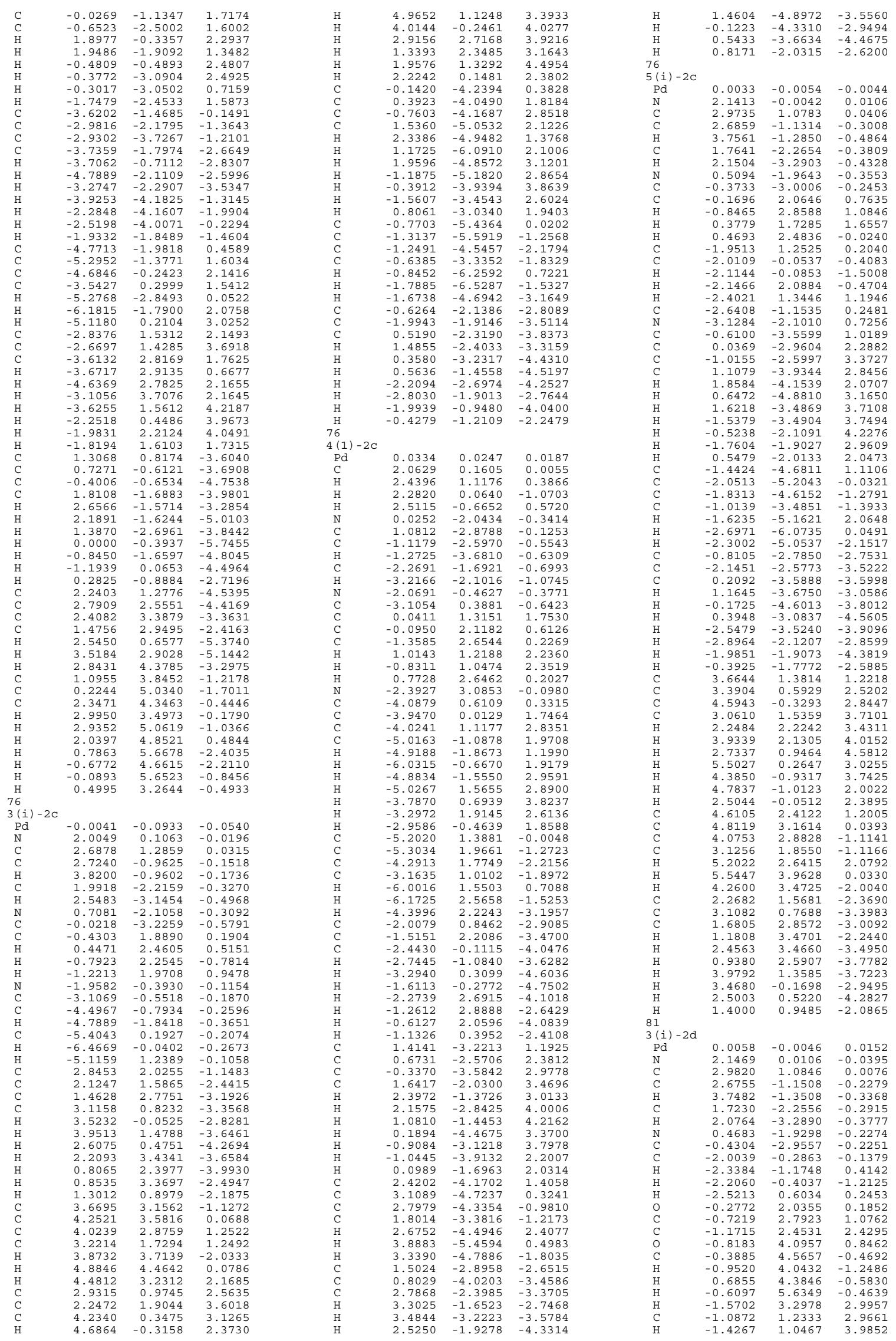

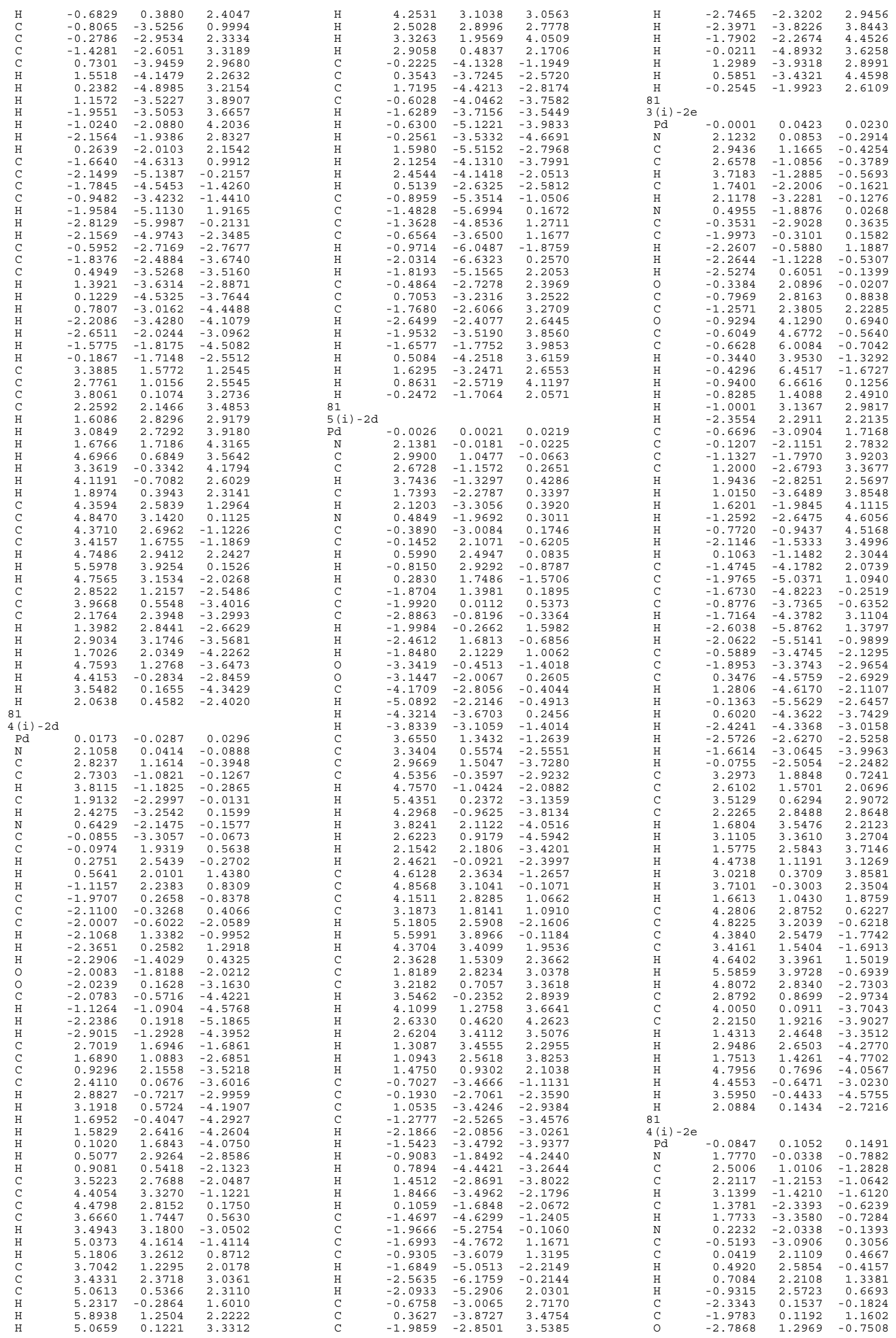

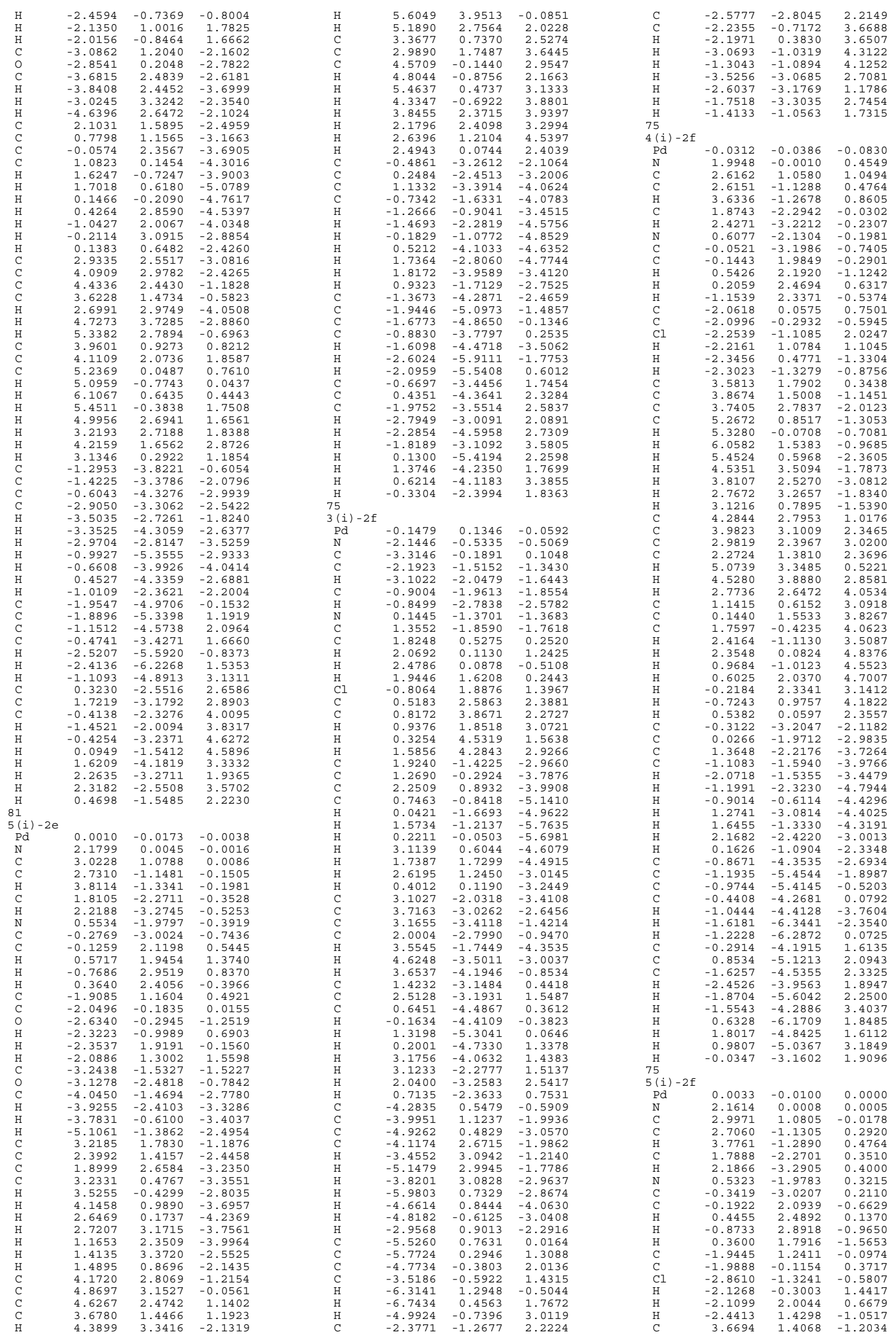

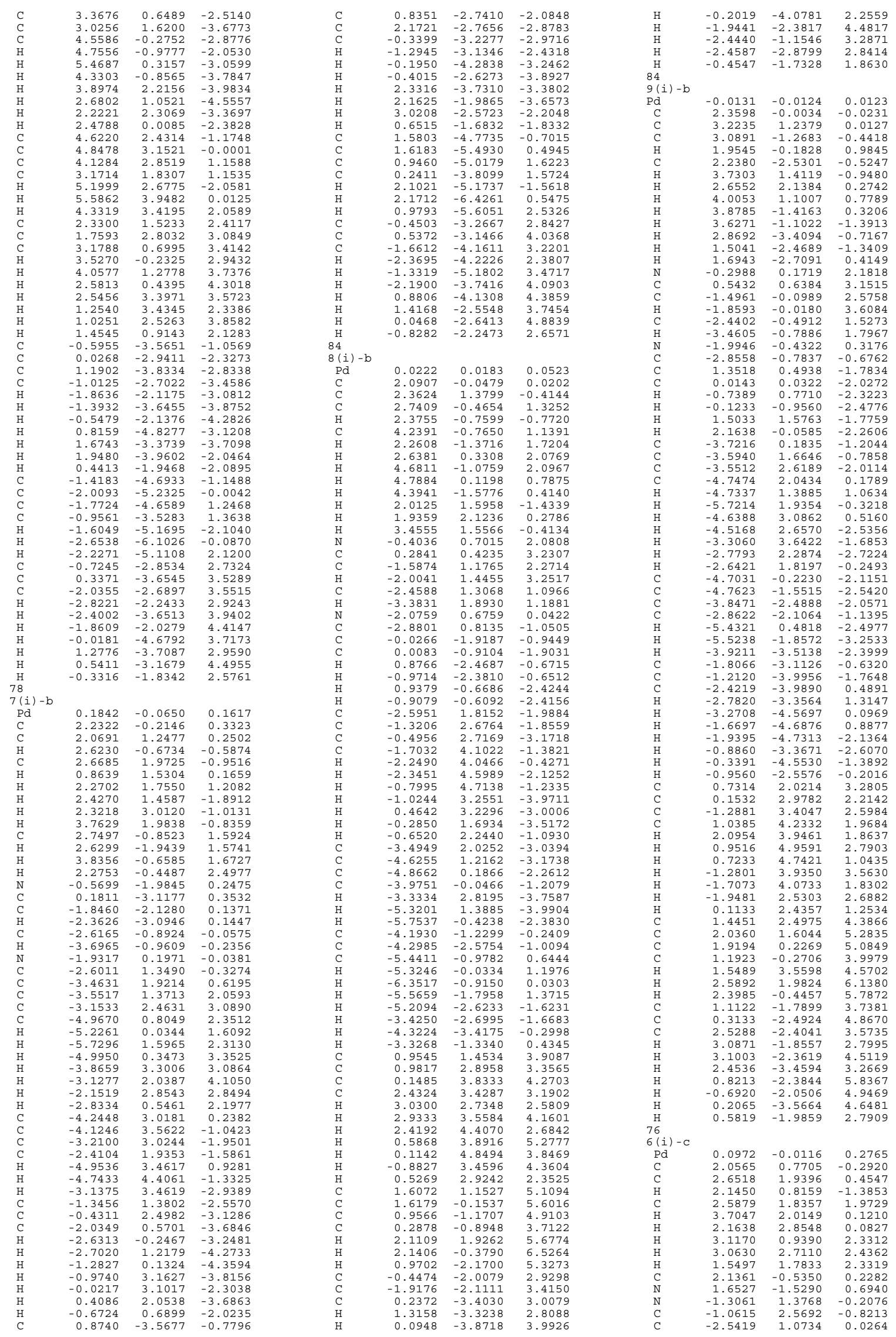

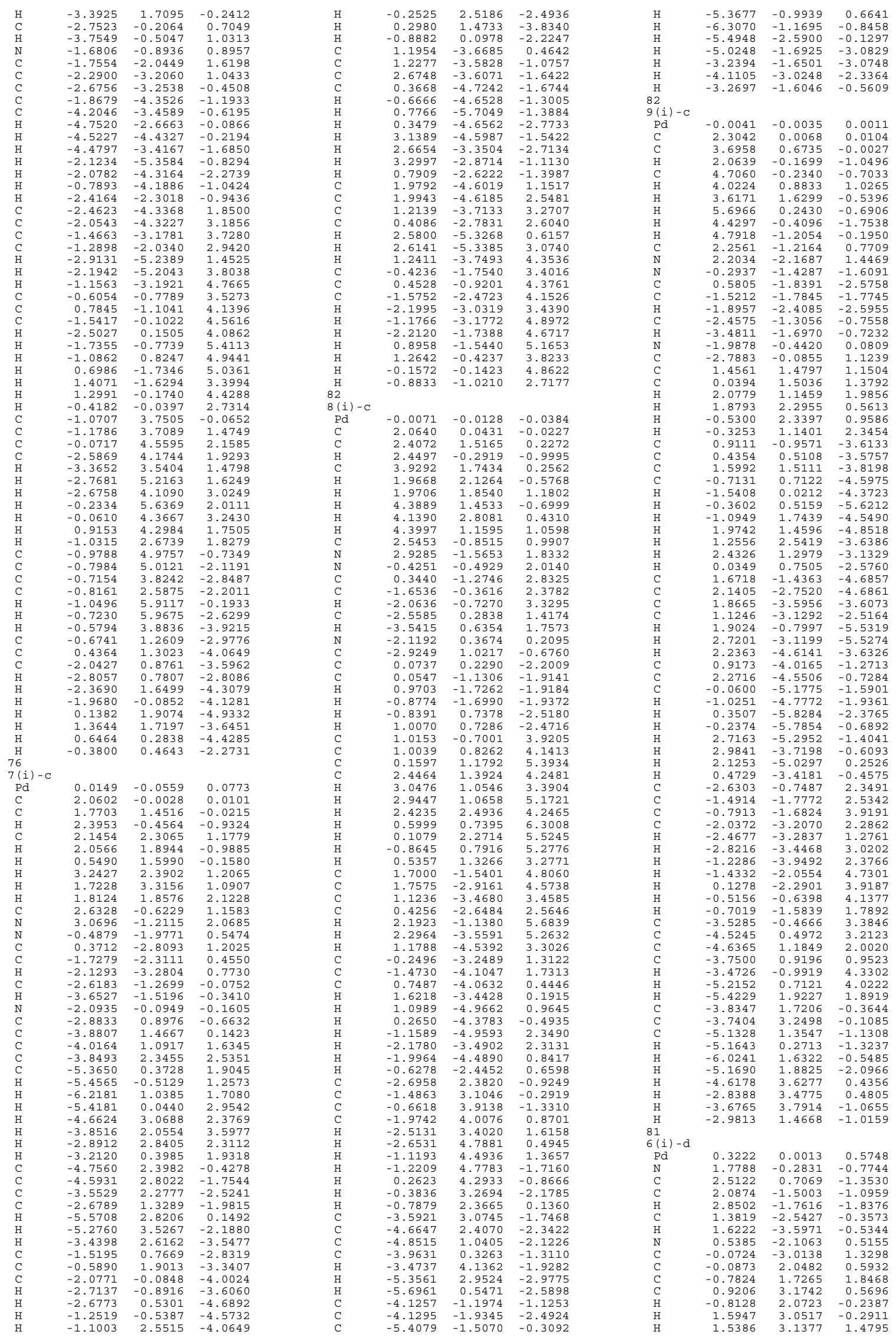

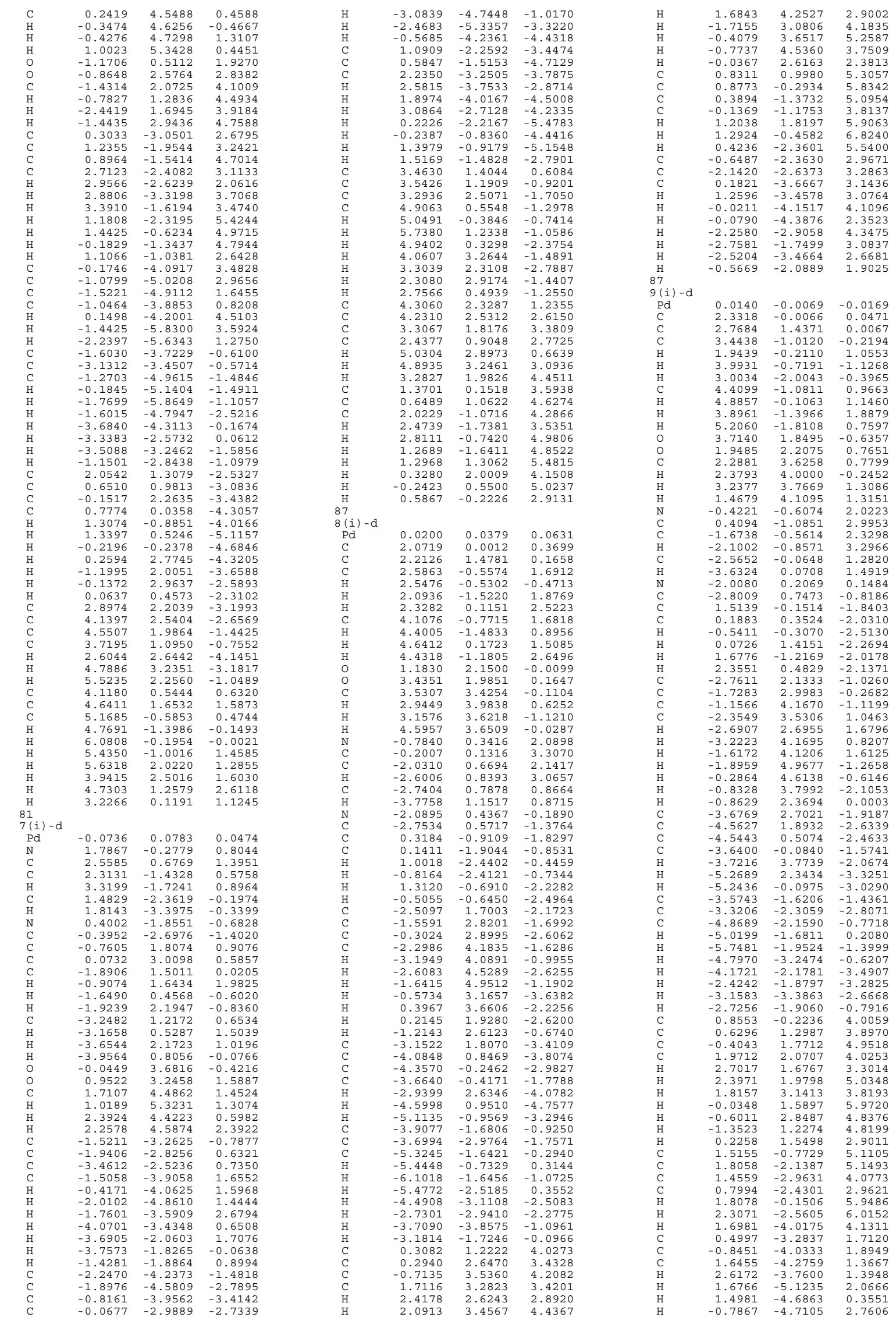

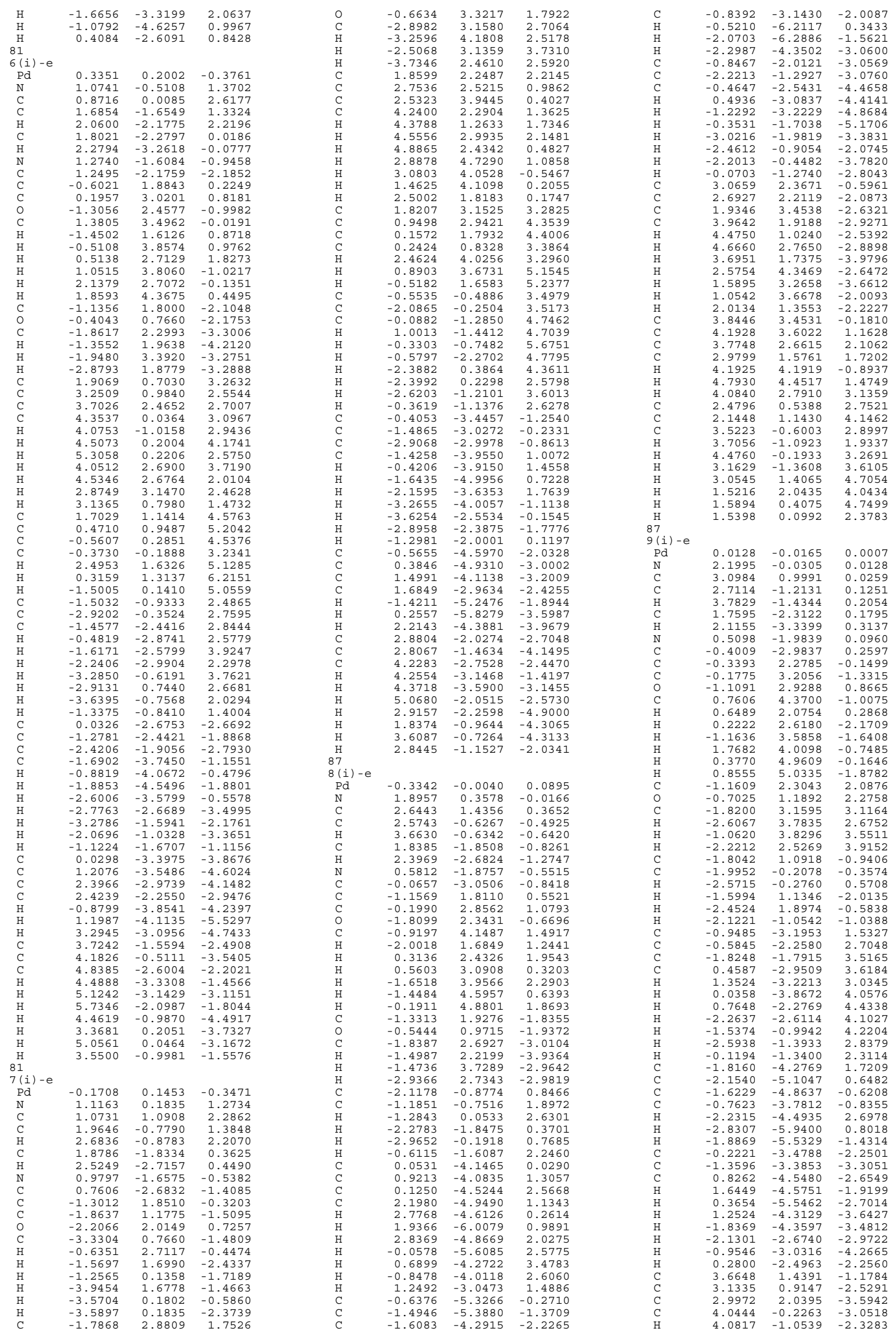

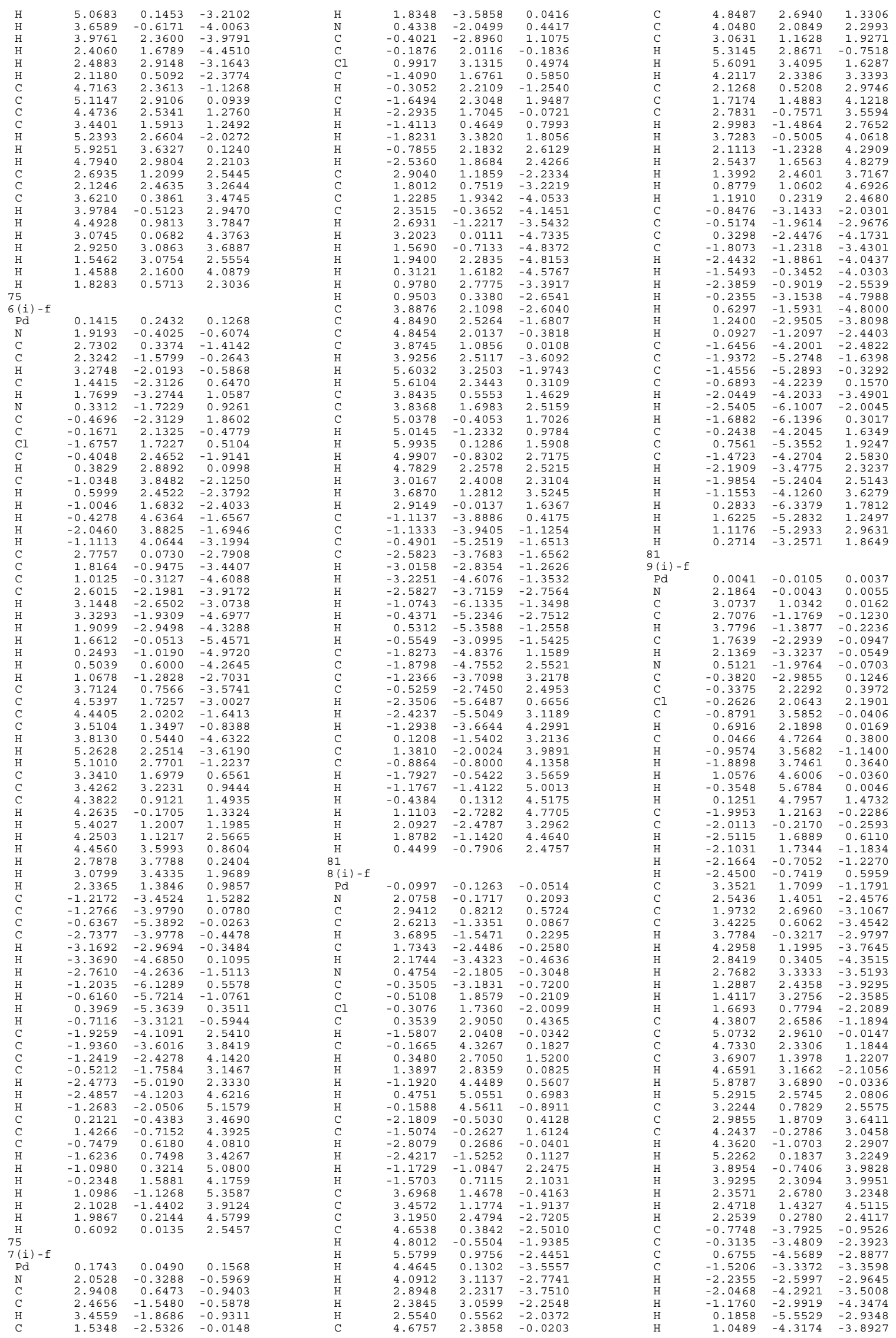


$\begin{array}{lrrr}\text { H } & 1.5353 & -4.6394 & -2.2042 \\ \mathrm{H} & 0.2153 & -2.5130 & -2.4164 \\ \mathrm{C} & -1.5994 & -4.8941 & -0.6979 \\ \mathrm{C} & -2.0643 & -5.1453 & 0.5948 \\ \mathrm{C} & -1.7059 & -4.3012 & 1.6480 \\ \mathrm{C} & -0.8809 & -3.1943 & 1.4183 \\ \mathrm{H} & -1.8871 & -5.5690 & -1.4958 \\ \mathrm{H} & -2.7064 & -6.0009 & 0.7816 \\ \mathrm{H} & -2.0743 & -4.5271 & 2.6411 \\ \mathrm{C} & -0.5067 & -2.2188 & 2.5579 \\ \mathrm{C} & 0.7330 & -2.7555 & 3.3193 \\ \mathrm{C} & -1.6780 & -1.9306 & 3.5389 \\ \mathrm{H} & -2.5902 & -1.6737 & 2.9793 \\ \mathrm{H} & -1.8909 & -2.7923 & 4.1877 \\ \mathrm{H} & -1.4220 & -1.0809 & 4.1920 \\ \mathrm{H} & 0.5030 & -3.7290 & 3.7786 \\ \mathrm{H} & 1.5820 & -2.8850 & 2.6312 \\ \mathrm{H} & 1.0331 & -2.0517 & 4.1115 \\ \mathrm{H} & -0.2384 & -1.2418 & 2.1233 \\ & & & \end{array}$



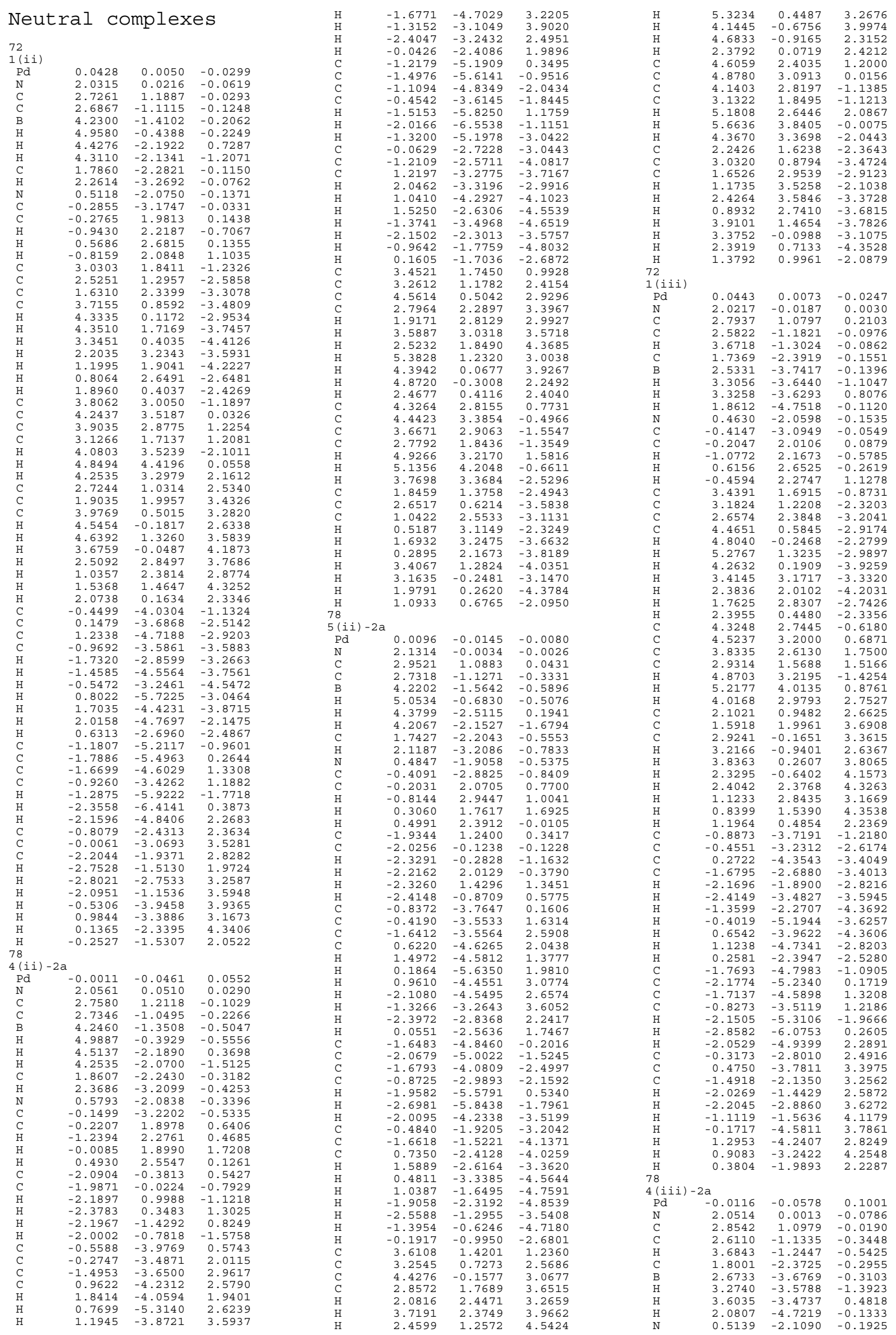

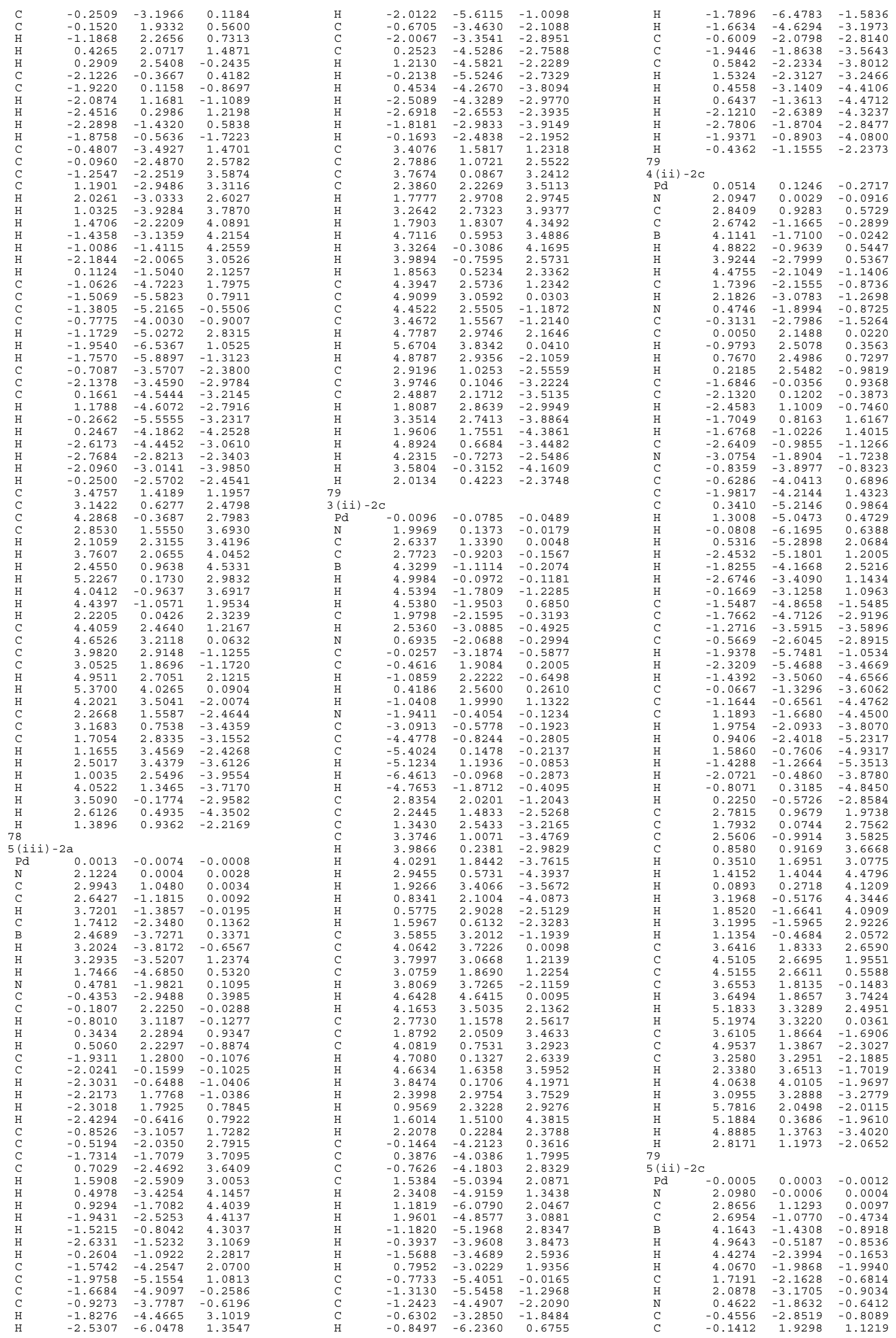

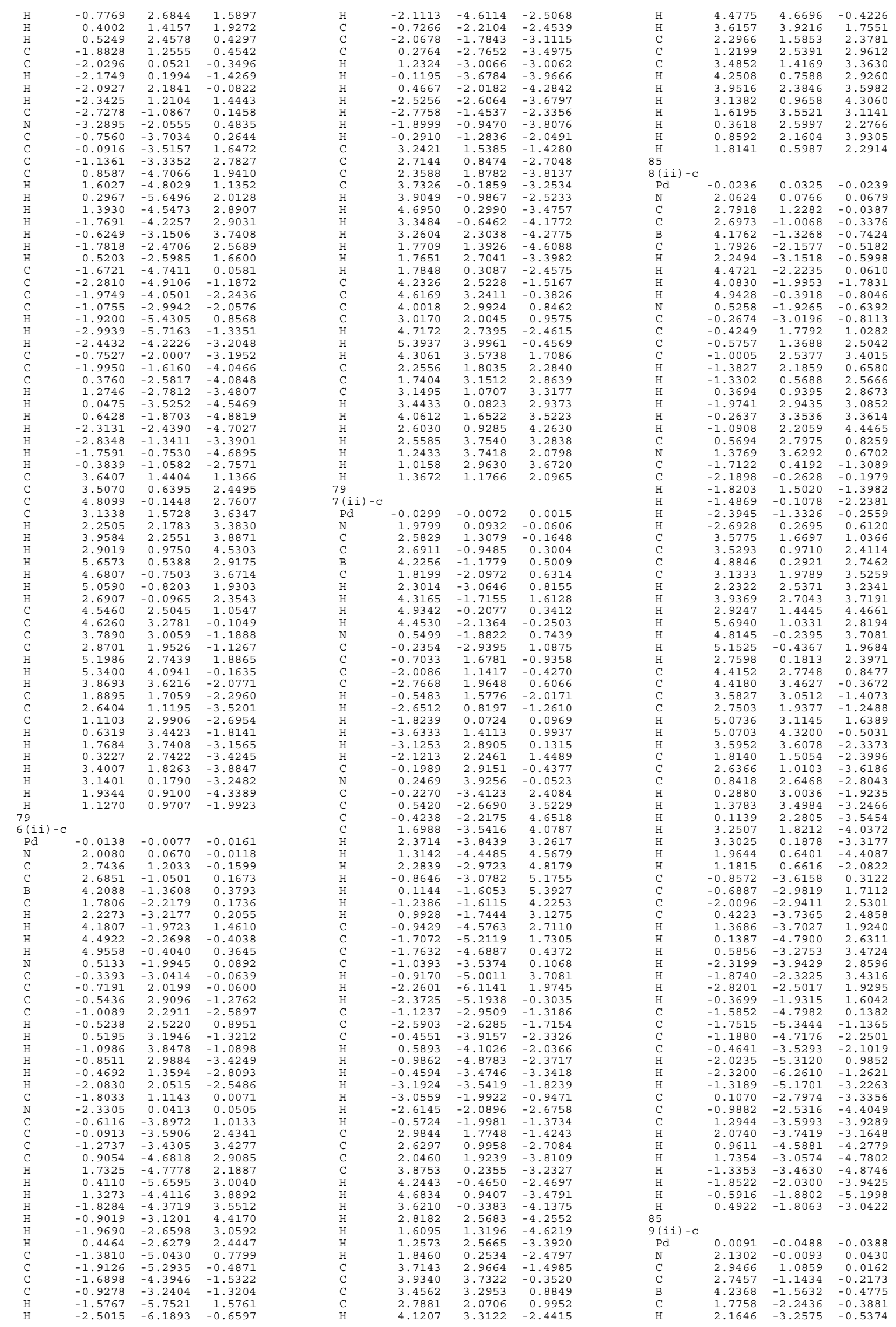

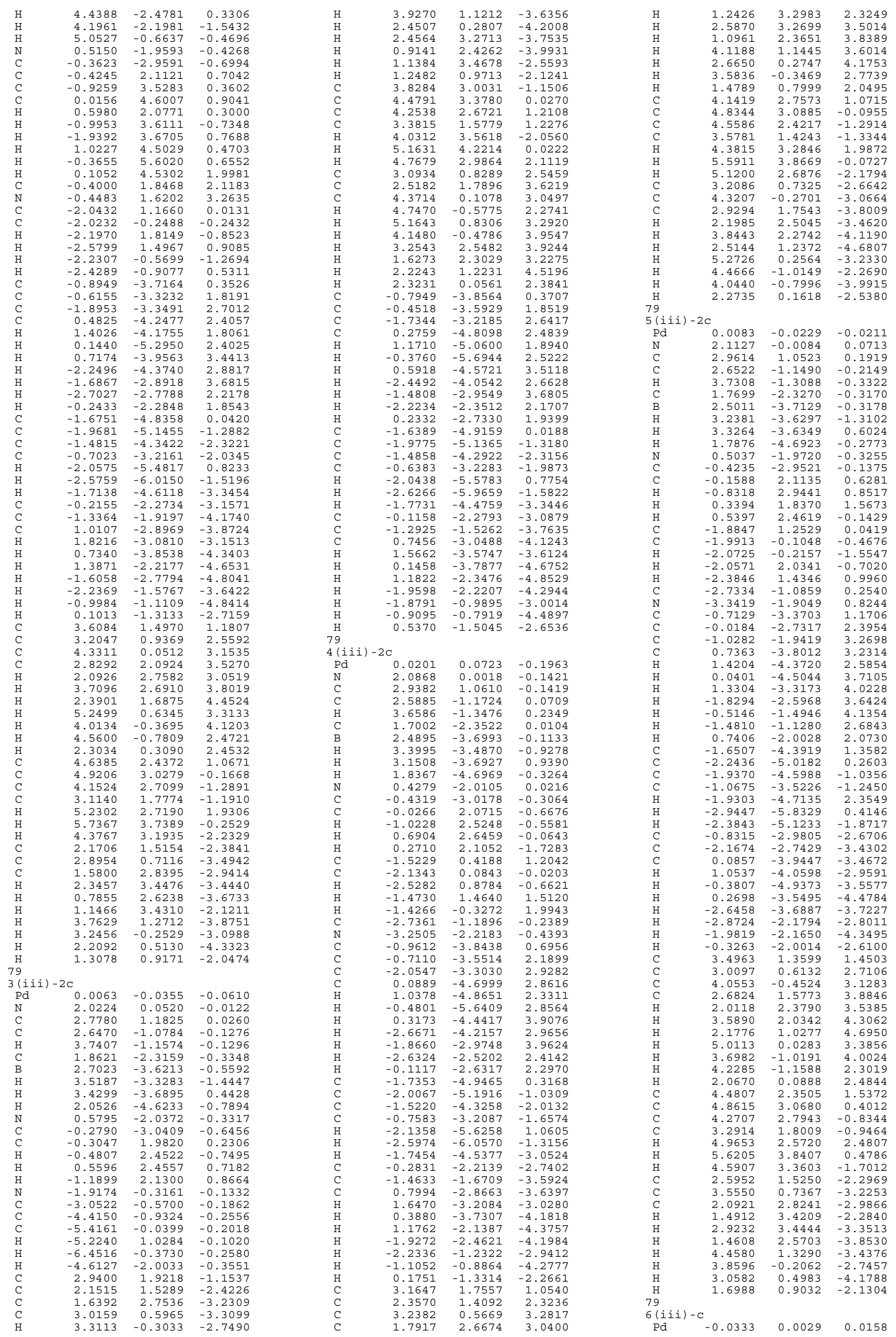

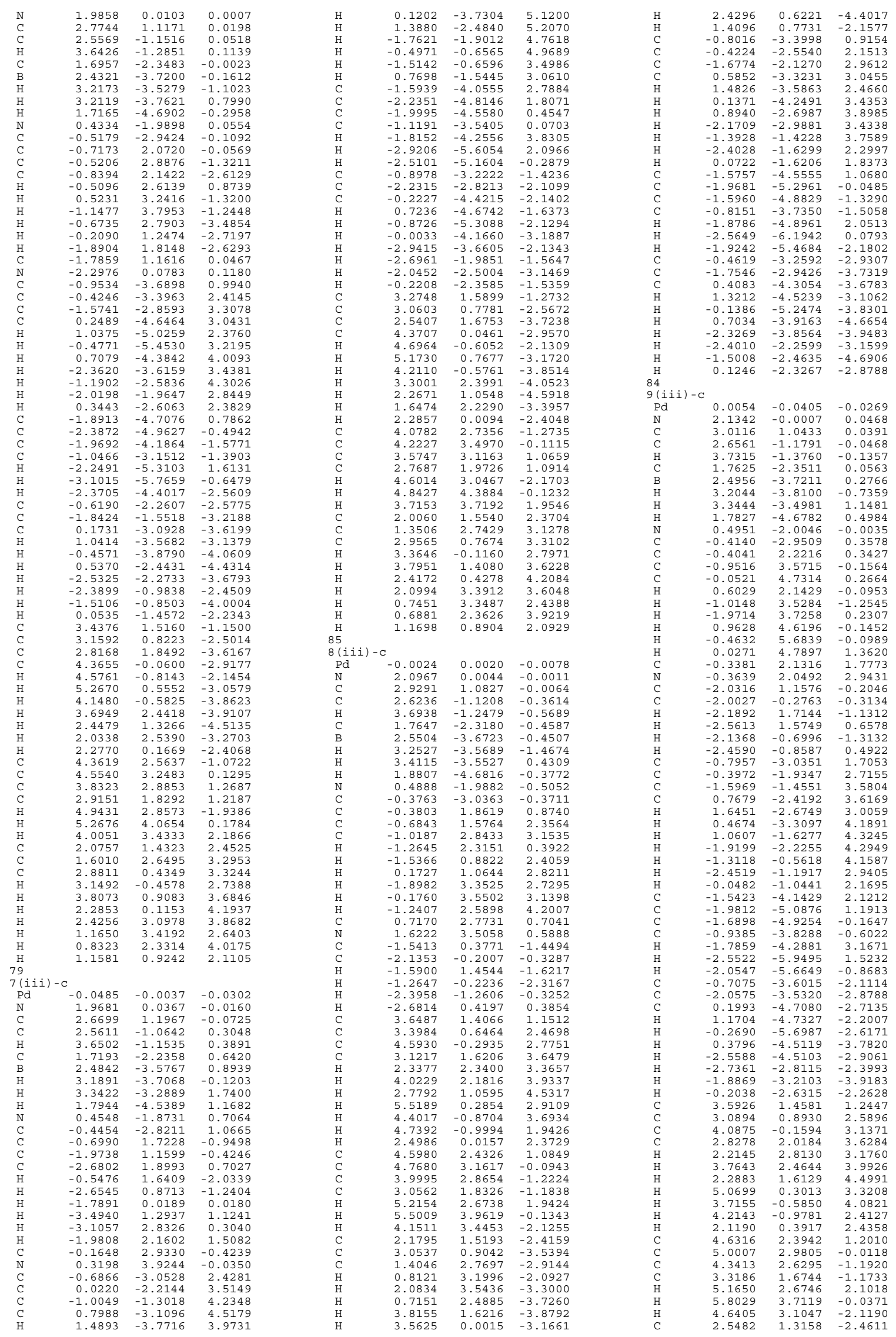


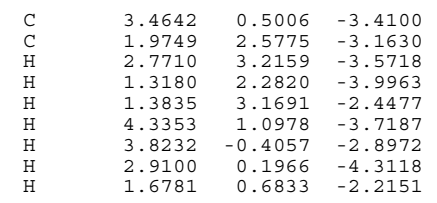



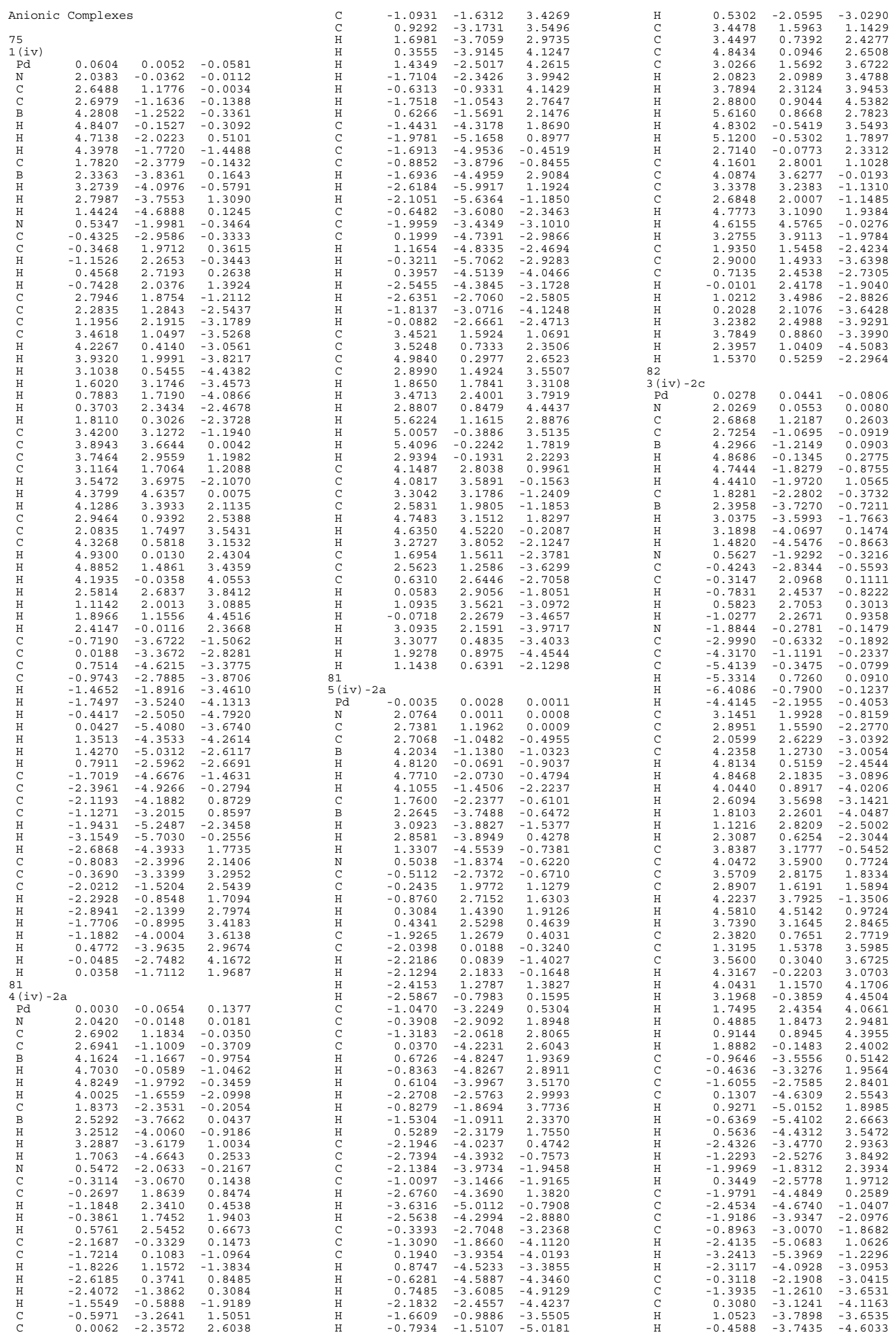

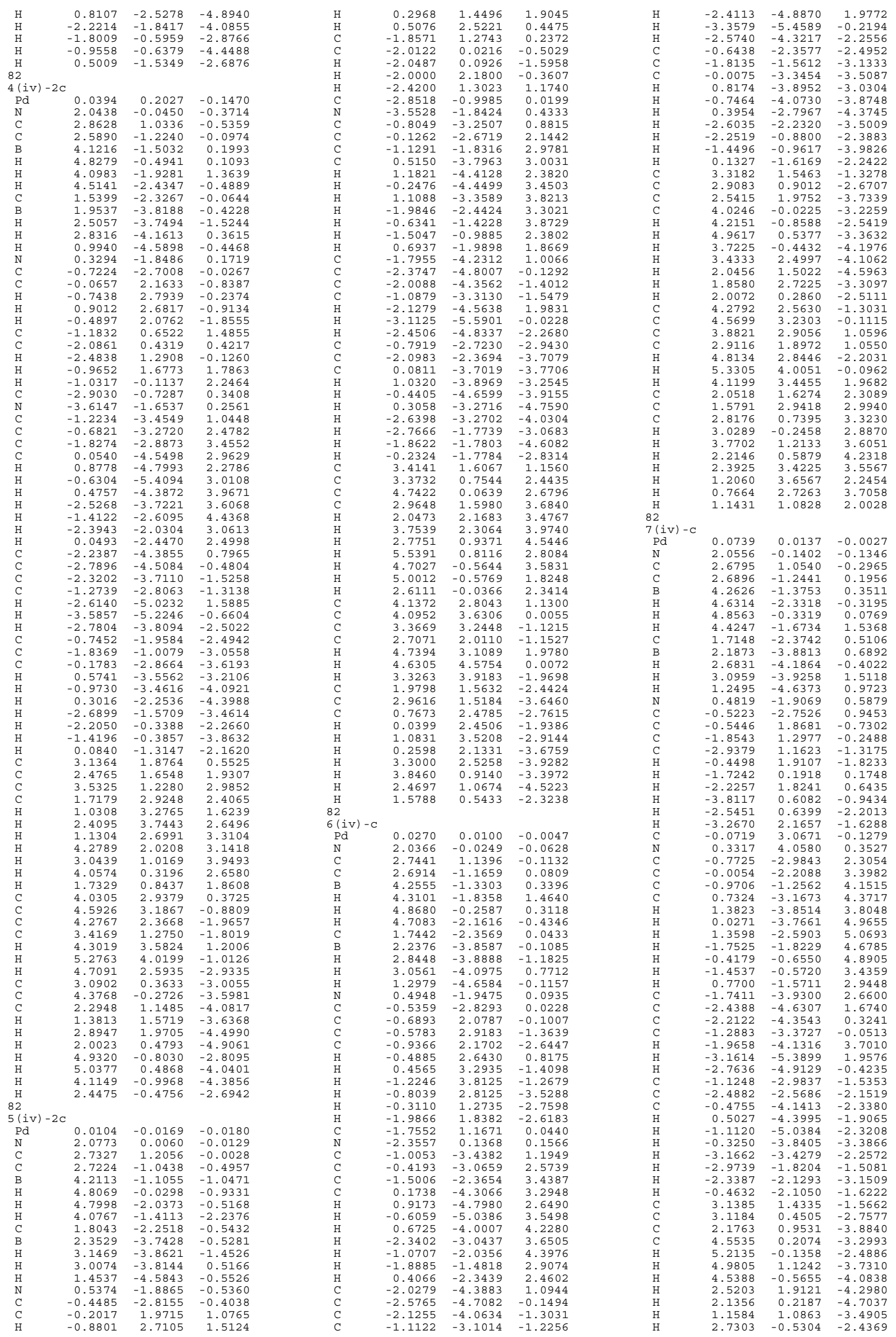

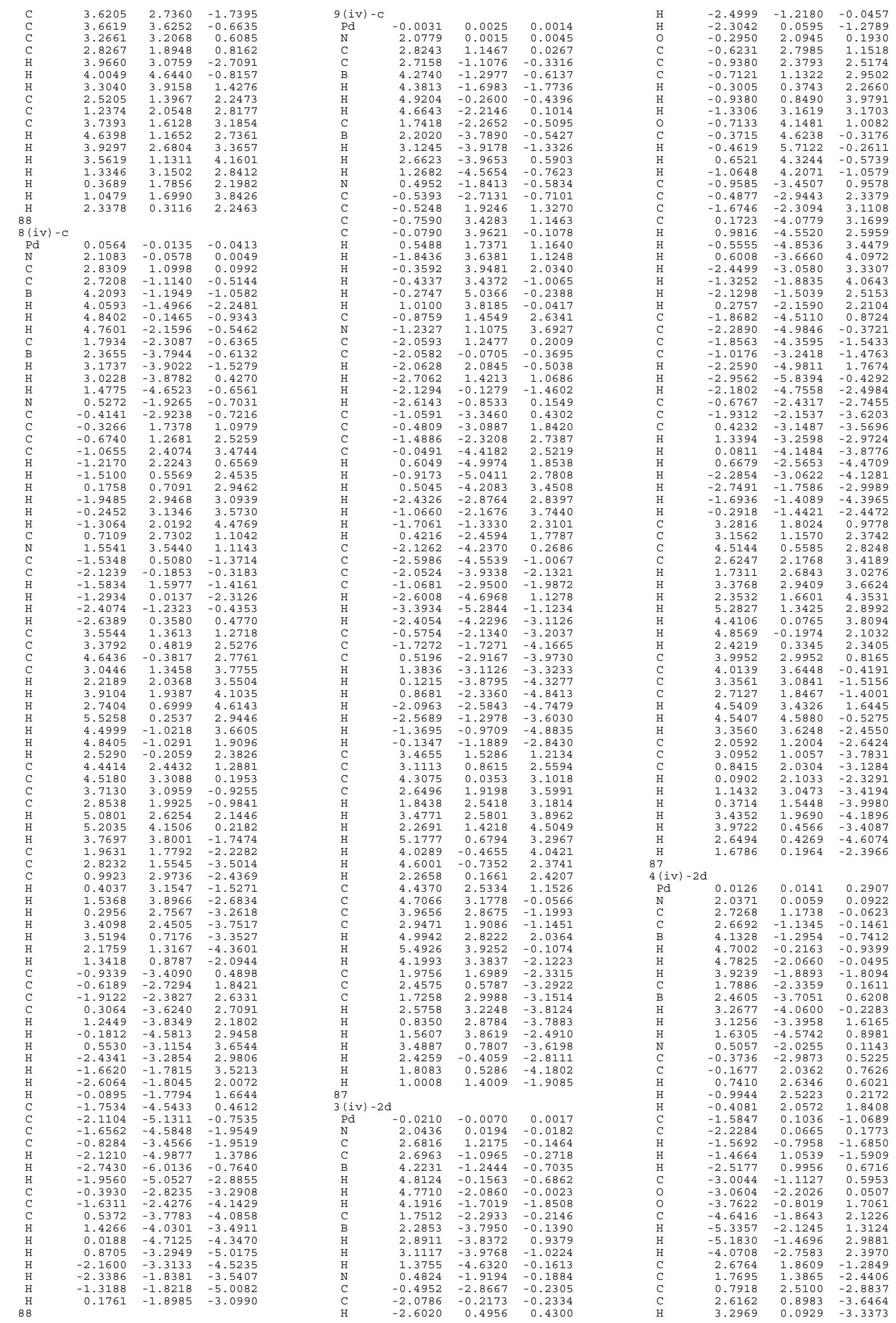

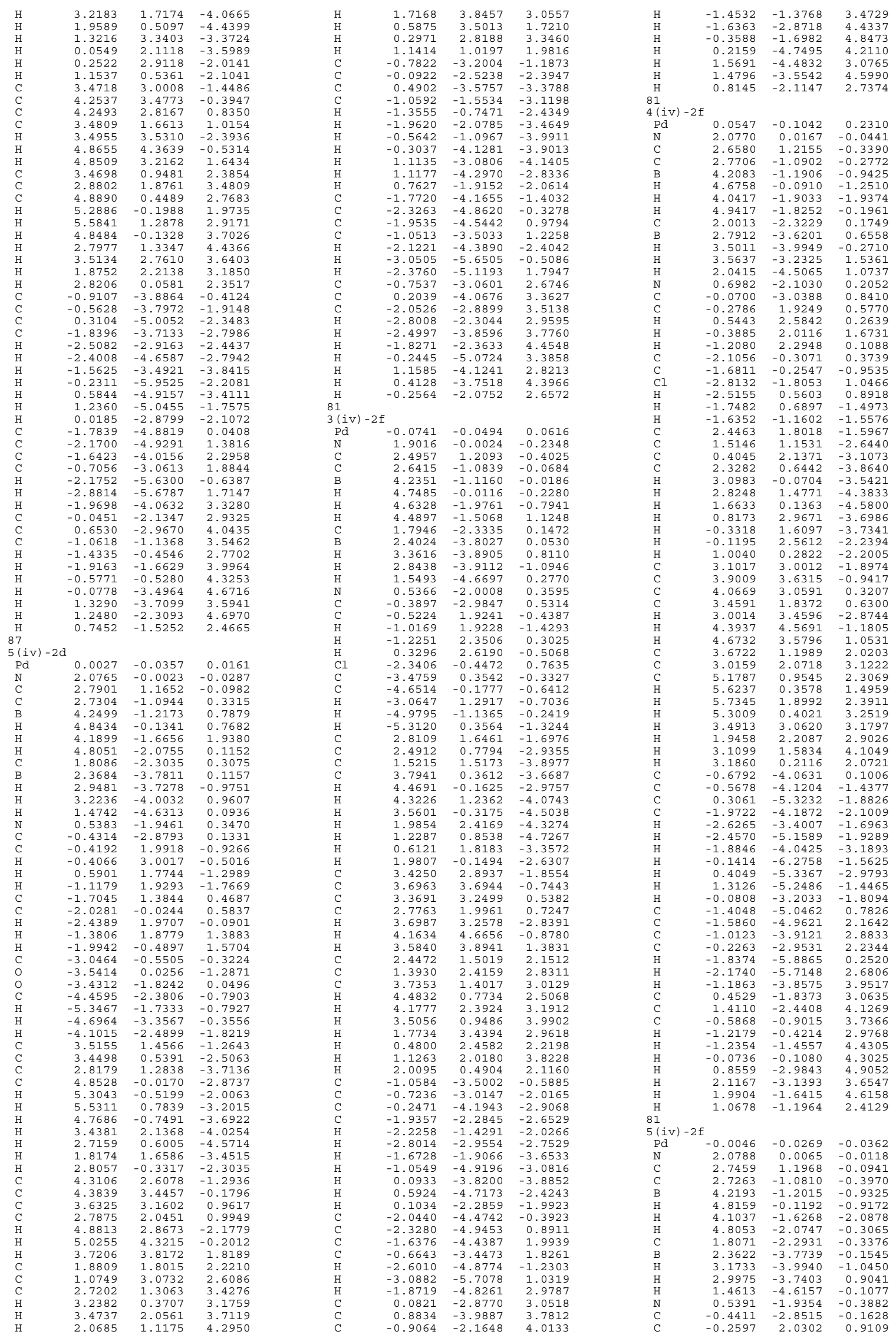


\begin{tabular}{|c|c|c|c|}
\hline $\mathrm{H}$ & -0.9463 & 2.8162 & 1.2383 \\
\hline $\mathrm{H}$ & 0.4944 & 2.5099 & 0.2754 \\
\hline $\mathrm{H}$ & 0.1850 & 1.5779 & 1.8080 \\
\hline $\mathrm{C}$ & -1.9019 & 1.2559 & 0.0798 \\
\hline C & -1.9758 & -0.0671 & -0.4991 \\
\hline $\mathrm{Cl}$ & -3.1343 & -1.2357 & 0.2942 \\
\hline $\mathrm{H}$ & -2.1158 & -0.1601 & -1.5791 \\
\hline $\mathrm{H}$ & -2.4909 & 1.3777 & 0.9931 \\
\hline $\mathrm{H}$ & -2.0232 & 2.0915 & -0.6192 \\
\hline $\mathrm{C}$ & 2.7334 & 1.9093 & -1.3038 \\
\hline C & 1.9903 & 1.3767 & -2.5512 \\
\hline C & 0.8077 & 2.3012 & -2.9476 \\
\hline $\mathrm{C}$ & 2.9676 & 1.1987 & -3.7459 \\
\hline $\mathrm{H}$ & 3.8292 & 0.5837 & -3.4484 \\
\hline $\mathrm{H}$ & 3.3429 & 2.1670 & -4.1072 \\
\hline 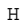 & 2.4577 & 0.6975 & -4.5838 \\
\hline $\mathrm{H}$ & 1.1582 & 3.3124 & -3.2004 \\
\hline $\mathrm{H}$ & 0.2797 & 1.8899 & -3.8223 \\
\hline $\mathrm{H}$ & 0.0879 & 2.3778 & -2.1210 \\
\hline $\mathrm{H}$ & 1.5571 & 0.3840 & -2.3452 \\
\hline C & 3.4203 & 3.1267 & -1.3717 \\
\hline C & 4.1514 & 3.5901 & -0.2761 \\
\hline $\mathrm{C}$ & 4.1696 & 2.8606 & 0.9141 \\
\hline C & 3.4255 & 1.6816 & 1.0330 \\
\hline $\mathrm{H}$ & 3.3990 & 3.7271 & -2.2739 \\
\hline $\mathrm{H}$ & 4.7071 & 4.5201 & -0.3499 \\
\hline $\mathrm{H}$ & 4.7695 & 3.2251 & 1.7399 \\
\hline $\mathrm{C}$ & 3.3586 & 0.9400 & 2.3858 \\
\hline $\mathrm{C}$ & $\begin{array}{l}2.9249 \\
2.920\end{array}$ & 1.8876 & $\begin{array}{l}3.5391 \\
3.530\end{array}$ \\
\hline $\mathrm{C}$ & 4.7210 & 0.2709 & 2.7062 \\
\hline $\mathrm{H}$ & 4.9971 & -0.4350 & 1.9097 \\
\hline $\mathrm{H}$ & 5.5166 & 1.0257 & 2.7941 \\
\hline $\mathrm{H}$ & 4.6601 & -0.2846 & 3.6551 \\
\hline $\mathrm{H}$ & 3.7039 & 2.6268 & 3.7746 \\
\hline $\mathrm{H}$ & 2.0063 & 2.4276 & 3.2656 \\
\hline $\mathrm{H}$ & 2.7264 & 1.3055 & 4.4529 \\
\hline $\mathrm{H}$ & 2.5975 & 0.1430 & 2.3374 \\
\hline $\mathrm{C}$ & -1.0792 & -3.4627 & -1.2514 \\
\hline C & -0.7693 & -3.0282 & -2.6998 \\
\hline $\mathrm{C}$ & 0.1190 & -4.0878 & -3.4021 \\
\hline C & -2.0658 & -2.7694 & -3.5180 \\
\hline $\mathrm{H}$ & -2.7483 & -2.1141 & -2.9569 \\
\hline $\mathrm{H}$ & -2.5962 & -3.7038 & -3.7511 \\
\hline $\mathrm{H}$ & -1.8189 & -2.2784 & -4.4727 \\
\hline $\mathrm{H}$ & -0.3959 & -5.0595 & -3.4427 \\
\hline $\mathrm{H}$ & 0.3525 & -3.7707 & -4.4304 \\
\hline $\mathrm{H}$ & 1.0651 & -4.2168 & -2.8571 \\
\hline $\mathrm{H}$ & -0.2162 & -2.0735 & -2.6860 \\
\hline $\mathrm{C}$ & -2.0112 & -4.4756 & -0.9999 \\
\hline C & -2.3898 & -4.7764 & 0.3099 \\
\hline 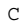 & -1.8045 & -4.1005 & 1.3823 \\
\hline C & -0.7943 & -3.1577 & 1.1605 \\
\hline $\mathrm{H}$ & -2.4521 & -5.0400 & -1.8131 \\
\hline $\mathrm{H}$ & -3.1419 & -5.5374 & 0.4949 \\
\hline $\mathrm{H}$ & -2.1477 & -4.3213 & 2.3864 \\
\hline $\mathrm{C}$ & -0.0933 & -2.4816 & 2.3621 \\
\hline C & 0.5036 & -3.5379 & 3.3324 \\
\hline C & -1.0584 & -1.5205 & 3.1049 \\
\hline $\mathrm{H}$ & -1.3821 & -0.7140 & 2.4311 \\
\hline $\mathrm{H}$ & -1.9478 & -2.0562 & 3.4681 \\
\hline $\mathrm{H}$ & -0.5514 & -1.0606 & 3.9676 \\
\hline $\mathrm{H}$ & -0.2834 & -4.1053 & 3.8494 \\
\hline $\mathrm{H}$ & 1.1374 & -4.2461 & 2.7775 \\
\hline $\mathrm{H}$ & 1.1234 & -3.0449 & 4. \\
\hline $\mathrm{H}$ & & -1.8645 & 2.0227 \\
\hline
\end{tabular}

